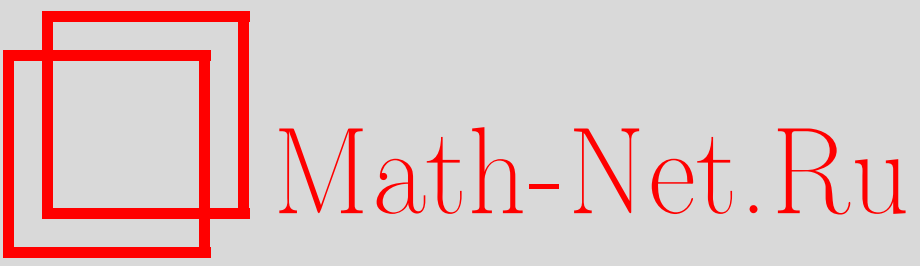

K. В. Степаньянц, Проблема аномалий в $N=1$ суперсимметричной электродинамике как следствие противоречивости метода размерной редукции, ТМФ, 2004, том 140, номер 1, 53-77

DOI: https://doi.org/10.4213/tmf83

Использование Общероссийского математического портала Math-Net.Ru подразумевает, что вы прочитали и согласны с пользовательским соглашением

http: //www . mathnet.ru/rus/agreement

Параметры загрузки:

IP : 18.209 .158 .208

26 апреля 2023 г., 18:18:55 
Том 140, № 1

июль, 2004

(C) 2004 r.

К.В. Степаньянц*

\section{ПРОБЛЕМА АНОМАЛИЙ В $N=1$ СУПЕРСИММЕТРИЧНОЙ ЭЛЕКТРОДИНАМИКЕ КАК СЛЕДСТВИЕ ПРОТИВОРЕЧИВОСТИ МЕТОДА РАЗМЕРНОЙ РЕДУКЦИИ}

Производится сравнение вычисления двухпетлевой $\beta$-функции в $N=1$ суперсимметричной электродинамике при использовании регуляризации высшими производными и регуляризации размерной редукцией. Показано, что перенормированное эффективное действие одинаково для обеих регуляризаций. Однако, в отличие от размерной редукции, $\beta$-фуункция, определенная как производная перенормированной константы связи по $\ln \mu$, в методе высших производных оказывается чисто однопетлевой. Поэтому проблема аномалий при использовании такой регуляризации не возникает. Это связано с тем, что в методе высших производных диаграммы с контрчленными вставками дают ненулевой вклад, который вычислен точно во всех порядках теории возмущений. При использовании размерной редукции этот вклад оказывается равным нулю. Приводятся аргументы в пользу того, что такой результат является следствием математической противоречивости размерной редукции и что именно он приводит к проблеме аномалий.

Ключевые слова: суперсимметрия, регуляризация, высшие ковариантные производные, размерная редукция.

\section{1. ВВЕДЕНИЕ}

Хорошо известно [1]-[4], что в суперсимметричных теориях аксиальная аномалия и аномалия следа тензора энергии-импульса принадлежат одному супермультиплету. В силу теоремы Адлера-Бардина [5], [6] аксиальная аномалия является чисто однопетлевой, тогда как аномалия следа пропорциональна $\beta$-функции [7]. Поэтому суперсимметричная инвариантность должна, по-видимому, приводить к тому, что в суперсимметричных теориях поправки к $\beta$-функции

$$
\beta=\frac{d}{d \ln \mu}\left(\frac{e^{2}}{4 \pi}\right),
$$

\footnotetext{
* Московский государственный университет, Москва, Россия. E-mail: stepan@theor.phys.msu.su
} 
где $e$ - перенормированная константа связи, должны сушествовать только в однопетлевом приближении [8]. Этот факт действительно имеет место в $N=2$ суперсимметричных теориях [9]. Однако вычисления, выполненные для $N=1$ суперсимметричных моделей в рамках метода размерной редукции [10]-[12], показали, что двухпетлевой вклад в $\beta$-функцию (1) оказывается отличным от нуля. Полученное противоречие в литературе получило название проблемы аномалий.

Предпринимались неоднократные попытки решения этой проблемы. Так, например, в работе [13] впервые было отмечено, что в $\beta$-функции может присутствовать вклад аномалии Кониши [14], [15]. Исследование этого вклада в работе [13], а также требование ренорминвариантности инстантонных вкладов [16] привели к построению так называемой точной $\beta$-функции Новикова-Шифмана-Вайнштейна-Захарова (НШВЗ), которая согласуется с двухпетлевыми вычислениями с использованием регуляризации при помощи размерной редукции. Тем не менее в трехпетлевом приближении [17]-[19] были выявлены разногласия между предсказаниями, следуюшими из точной $\beta$-функции, и конкретными вычислениями. При этом было показано [18], что эти разногласия могут быть устранены специальным выбором схемы перенормировки. В принципе схему, в которой получается точная $\beta$-функция, можно построить, устанавливая ее связь с $\overline{\mathrm{MS}}$-схемой в каждом порядке теории возмущений [20].

Простое и красивое решение проблемы аномалий, отличное от решения, предложенного в [13], было дано в работе [21], основная идея которой заключается в том, что высшие поправки в $\beta$-функцию НШВЗ появляются благодаря аномальному якобиану, который возникает при переходе от голоморфной к канонической нормировке суперполей. В случае суперсимметричной электродинамики голоморфная нормировка означает, что перенормированное действие записывается в виде

$$
\begin{aligned}
S_{\text {ren }}= & \frac{1}{4 e^{2}} Z_{3}\left(e, \frac{\Lambda}{\mu}\right) \operatorname{Re} \int d^{4} x d^{2} \theta W_{a} C^{a b} W_{b}+ \\
& +\frac{1}{4} Z\left(e, \frac{\Lambda}{\mu}\right) \int d^{4} x d^{4} \theta\left(\phi^{*} e^{2 V} \phi+\tilde{\phi}^{*} e^{-2 V} \tilde{\phi}\right),
\end{aligned}
$$

тогда как в канонической нормировке

$$
\begin{aligned}
S_{\text {ren }}= & \frac{1}{4 e^{2}} Z_{3}\left(e, \frac{\Lambda}{\mu}\right) \operatorname{Re} \int d^{4} x d^{2} \theta W_{a} C^{a b} W_{b}+ \\
& +\frac{1}{4} \int d^{4} x d^{4} \theta\left(\phi^{*} e^{2 V} \phi+\tilde{\phi}^{*} e^{-2 V} \tilde{\phi}\right) .
\end{aligned}
$$

Было высказано предположение, что в первом случае $\beta$-функция является чисто однопетлевой, а во втором совпадает с результатом НШВЗ. В принципе это решение проблемы аномалий отличается от решения Шифмана и Вайнштейна. Кроме того, оно противоречит явным двухпетелевым вычислениям, сделанным при помоши регуляризации 
размерной редукцией. В работе [21] было высказано предположение, что чисто однопетлевой результат может возникать при использовании регуляризации высшими ковариантными производными [22], [23], дополненной регуляризацией Паули-Вилларса для устранения остаточных однопетлевых расходимостей. Известно, что на однопетлевом уровне такая регуляризация всегда согласуется с результатом вычислений методом размерной регуляризации (редукции) [24]. Конкретные двухпетлевые вычисления $\beta$-функции (1), а также двухпетлевой аномальной размерности при использовании регуляризации высшими производными были впервые выполнены в работах [25], [26] для $N=1$ суперсимметричной электродинамики. При этом для двухпетлевого вклада в $\beta$-функцию был получен нулевой результат, который действительно демонстрирует отсутствие проблемы аномалий в свете решения, предложенного в работе [21]. Тем не менее по-прежнему непонятым остается вопрос о том, почему методы размерной редукции и высших производных приводят к различным результатам для схемно-независимой двухпетлевой $\beta$-функции. В работе [25] было замечено, что по сравнению с методом размерной редукции в методе высших производных появляется нетривиальный вклад диаграмм со вставкой однопетлевых контрчленов. Однако аккуратный анализ этого вклада выполнен не был.

В этой работе вклад диаграмм с контрчленными вставками детально анализируется с использованием различных методов регуляризации. При этом наибольшее внимание уделяется сравнению методов размерной редукции [27] и высших производных, которые явно сохраняют суперсимметрию теории. Очень важно заметить, что уже вскоре после того, как была предложена регуляризация с помошью размерной редукции, было показано, что эта регуляризация противоречива [28]. Следовательно, непосредственное применение размерной редукции к вычислению, например, треугольной диаграммы, определяюшей аксиальную аномалию, приводит к нулевому результату, поскольку размерная редукция сохраняет киральную симметрию. При этом необходимо подчеркнуть сушественную разницу между размерной регуляризацией [29] и размерной редукцией, поскольку в рамках размерной регуляризации аксиальная аномалия может быть легко вычислена [29]. В принципе можно вычислять аномалии и с помошью размерной редукции, если накладьвать некоторые заведомо противоречивые условия, например полагая $\operatorname{tr}(A B) \neq \operatorname{tr}(B A)$ [30] или требуя в случае $n<4$ выполнения некоторых тождеств меж ду $\gamma$-матрицами, справедливых только при $n>4$ [31] (в методе размерной редукции размерность пространства $n$ с необходимостью должна выбираться меньше 4 [27]).

В настоящей работе утверждается, что отмеченные противоречия метода размерной редукции приводят к противоречию между структурой мультиплета аномалий и результатом для $\beta$-функции (1). Соответствуюшие аргументы поясняются сравнением вычисления двухпетлевой $\beta$-функции $N=1$ суперсимметричной электродинамики с аналогичным вычислением с использованием метода высших производных.

Статья организована следуюшим образом. Основные сведения об $N=1$ суперсимметричной электродинамике и различных способах введения в нее регуляризации на- 
поминаются в разделе 2. Здесь же указаны основные противоречия метода размерной редукции. Анализ двухпетлевого вклада в $\beta$-функцию, его связь с аномалией Кониши и сравнение различных методов регуляризации приводятся в разделе 3 . Вычисление точной во всех порядках теории возмущений суммы диаграмм с контрчленными вставками при помоши метода высших производных выполнено в разделе 4. Полученные результаты обсуждаются в разделе 5 , а в приложение вынесены технические детали вычислений.

\section{2. СУПЕРСИММЕТРИЧНАЯ ЭЛЕКТРОДИНАМИКА И МЕТОДЫ ЕЕ РЕГУЛЯРИЗАЦИИ}

2.1. $N=1$ суперсимметричная электродинамика. $N=1$ суперсимметричная электродинамика в суперпространстве описьвается действием

$$
S_{0}=\frac{1}{4 e^{2}} \operatorname{Re} \int d^{4} x d^{2} \theta W_{a} C^{a b} W_{b}+\frac{1}{4} \int d^{4} x d^{4} \theta\left(\phi^{*} e^{2 V} \phi+\tilde{\phi}^{*} e^{-2 V} \tilde{\phi}\right) .
$$

При этом $\phi$ и $\tilde{\phi}$ - киральные суперполя, которые в терминах компонентных полей записываются как

$$
\begin{aligned}
& \phi(y, \theta)=\varphi(y)+\bar{\theta}\left(1+\gamma_{5}\right) \psi(y)+\frac{1}{2} \bar{\theta}\left(1+\gamma_{5}\right) \theta f(y), \\
& \tilde{\phi}(y, \theta)=\tilde{\varphi}(y)+\bar{\theta}\left(1+\gamma_{5}\right) \tilde{\psi}(y)+\frac{1}{2} \bar{\theta}\left(1+\gamma_{5}\right) \theta \tilde{f}(y),
\end{aligned}
$$

где $y^{\mu}=x^{\mu}+i \bar{\theta} \gamma^{\mu} \gamma_{5} \theta / 2$ - киральные координаты. При этом майорановские спиноры $\psi$ и $\tilde{\psi}$ могут быть объединены в один дираковский спинор

$$
\Psi=\frac{1}{\sqrt{2}}\left(\left(1+\gamma_{5}\right) \psi+\left(1-\gamma_{5}\right) \tilde{\psi}\right)
$$

Через $V$ в действии (4) обозначено абелево вешественное суперполе, которое в компонентах имеет вид

$$
\begin{aligned}
V(x, \theta)= & C(x)+i \sqrt{2} \bar{\theta} \gamma_{5} \xi(x)+\frac{1}{2}(\bar{\theta} \theta) K(x)+\frac{i}{2}\left(\bar{\theta} \gamma_{5} \theta\right) H(x)+\frac{1}{2}\left(\bar{\theta} \gamma^{\mu} \gamma_{5} \theta\right) A_{\mu}(x)+ \\
& +\sqrt{2}(\bar{\theta} \theta) \bar{\theta}\left(i \gamma_{5} \chi(x)+\frac{1}{2} \gamma^{\mu} \gamma_{5} \partial_{\mu} \xi(x)\right)+\frac{1}{4}(\bar{\theta} \theta)^{2}\left(D(x)-\frac{1}{2} \partial^{2} C(x)\right)
\end{aligned}
$$

где, в частности, $A_{\mu}$ представляет собой абелево калибровочное поле. Суперполе $W_{a}$ является суперсимметричным аналогом тензора напряженности калибровочного поля и в абелевом случае определяется как

$$
W_{a}=\frac{1}{16} \bar{D}\left(1-\gamma_{5}\right) D\left[\left(1+\gamma_{5}\right) D_{a} V\right]
$$

где суперсимметричная ковариантная производная $D$ записывается в виде

$$
D=\frac{\partial}{\partial \bar{\theta}}-i \gamma^{\mu} \theta \partial_{\mu}
$$


2.2. Регуляризация высшими производными. Для того чтобы регуляризовать модель (4) при помоши метода высших ковариантных производных, ее действие модифицируется следуюшим образом:

$$
\begin{aligned}
S_{0} \rightarrow S= & S_{0}+S_{\Lambda}=\frac{1}{4 e^{2}} \operatorname{Re} \int d^{4} x d^{2} \theta W_{a} C^{a b}\left(1+\frac{\partial^{2 n}}{\Lambda^{2 n}}\right) W_{b}+ \\
& +\frac{1}{4} \int d^{4} x d^{4} \theta\left(\phi^{*} e^{2 V} \phi+\tilde{\phi}^{*} e^{-2 V} \tilde{\phi}\right) .
\end{aligned}
$$

Заметим, что в силу абелевости рассматриваемой модели суперполе $W^{a}$ является калибровочно-инвариантным, благодаря чему регуляризуюшее слагаемое будет содержать обычные, а не ковариантные производные.

Квантование модели (10) производится полностью аналогично квантованию нерегуляризованной теории, подробно описанному в книге [32], и здесь не обсуждается. Отметим только, что калибровочную инвариантность удобно фиксировать добавлением слагаемых

$$
S_{\mathrm{gf}}=-\frac{1}{64 e^{2}} \int d^{4} x d^{4} \theta\left(V D^{2} \bar{D}^{2}\left(1+\frac{\partial^{2 n}}{\Lambda^{2 n}}\right) V+V \bar{D}^{2} D^{2}\left(1+\frac{\partial^{2 n}}{\Lambda^{2 n}}\right) V\right)
$$

где

$$
D^{2} \equiv \frac{1}{2} \bar{D}\left(1+\gamma_{5}\right) D, \quad \bar{D}^{2} \equiv \frac{1}{2} \bar{D}\left(1-\gamma_{5}\right) D,
$$

поскольку после добавления таких членов кинетический член калибровочного поля записывается в наиболее простом виде:

$$
S_{\text {gauge }}+S_{\text {gf }}=\frac{1}{4 e^{2}} \int d^{4} x d^{4} \theta V \partial^{2}\left(1+\frac{\partial^{2 n}}{\Lambda^{2 n}}\right) V .
$$

В силу абелевости исследуемой модели диаграммы с духовыми петлями отсутствуют.

Тем не менее введение слагаемого с высшими производными не приводит к полному устранению расходимостей. Действительно, индекс расходимости для модели (10) оказывается равным (см., например, [25])

$$
\omega_{\Lambda}=2-2 n(L-1)-E_{\phi}(n+1),
$$

где $L$ - количество петель, а $E_{\phi}$ - число внешних $\phi$-линий. Формула (14) означает, что даже при $n \geqslant 2$ в однопетлевых диаграммах по-прежнему сушествуют остаточные расходимости, для удаления которых необходимо добавить в производяший функционал детерминанты Паули-Вилларса [6]:

$$
\begin{aligned}
Z= & \int D V D \phi D \tilde{\phi} \prod_{i}\left(\operatorname{det} P V\left(V, M_{i}\right)\right)^{c_{i}} \exp \left\{i \left[\frac{1}{4 e^{2}} \int d^{4} x d^{4} \theta V \partial^{2}\left(1+\frac{\partial^{2 n}}{\Lambda^{2 n}}\right) V-\right.\right. \\
& -\frac{1}{4 e^{2}}\left(Z_{3}\left(e, \frac{\Lambda}{\mu}\right)-1\right) \int d^{4} x d^{4} \theta V \Pi_{1 / 2} \partial^{2}\left(1+\frac{\partial^{2 n}}{\Lambda^{2 n}}\right) V+ \\
& +\frac{1}{4} Z\left(e, \frac{\Lambda}{\mu}\right) \int d^{4} x d^{4} \theta\left(\phi^{*} e^{2 V} \phi+\tilde{\phi}^{*} e^{-2 V} \tilde{\phi}\right)+ \\
& \left.\left.+\int d^{4} x d^{4} \theta J V+\int d^{4} x d^{2} \theta(j \phi+\tilde{j} \tilde{\phi})+\int d^{4} x d^{2} \bar{\theta}\left(j^{*} \phi^{*}+\tilde{j}^{*} \tilde{\phi}^{*}\right)\right]\right\},
\end{aligned}
$$


где

$$
\begin{aligned}
(\operatorname{det} P V(V, M))^{-1}= & \int D \Phi D \tilde{\Phi} \exp \left\{i \left[Z\left(e, \frac{\Lambda}{\mu}\right) \frac{1}{4} \int d^{4} x d^{4} \theta\left(\Phi^{*} e^{2 V} \Phi+\tilde{\Phi}^{*} e^{-2 V} \tilde{\Phi}\right)+\right.\right. \\
& \left.\left.+\frac{1}{2} \int d^{4} x d^{2} \theta M \tilde{\Phi} \Phi+\frac{1}{2} \int d^{4} x d^{2} \bar{\theta} M \tilde{\Phi}^{*} \Phi^{*}\right]\right\},
\end{aligned}
$$

а коэффициенты $c_{i}$ удовлетворяют уравнениям

$$
\sum_{i} c_{i}=1, \quad \sum_{i} c_{i} M_{i}^{2}=0
$$

Далее мы будем предполагать, что $M_{i}=a_{i} \Lambda$, где $a_{i}$ - некоторые постоянные. Добавление детерминантов Паули-Вилларса позволяет сократить остаточные расходимости во всех однопетлевых диаграммах. Важно отметить, что величина $Z(e, \Lambda / \mu)$ в формулах (15) и (16) является одной и той же. Это необходимо для того, чтобы регуляризовать расходимости в диаграммах с контрчленными вставками, которые не содержат внутренних фотонных линий. Диаграммы, которые содержат петли полей Паули-Вилларса с внутренними фотонными линиями, в сумме являются конечными. Это проверено явно в двухпетлевом [25] и трехпетлевом [33] приближениях, а также может быть доказано во всех петлях.

При построении формулы (15) также было учтено, что благодаря суперсимметричной калибровочной инвариантности

$$
V \rightarrow V-\frac{1}{2}\left(A+A^{+}\right), \quad \phi \rightarrow e^{A} \phi, \quad \tilde{\phi} \rightarrow e^{-A} \tilde{\phi}
$$

где $A$ - произвольное скалярное киральное суперполе, перенормированное действие рассматриваемой модели может быть представлено как

$$
\begin{aligned}
S_{\text {ren }}= & \frac{1}{4 e^{2}} Z_{3}\left(e, \frac{\Lambda}{\mu}\right) \operatorname{Re} \int d^{4} x d^{2} \theta W_{a} C^{a b}\left(1+\frac{\partial^{2 n}}{\Lambda^{2 n}}\right) W_{b}+ \\
& +Z\left(e, \frac{\Lambda}{\mu}\right) \frac{1}{4} \int d^{4} x d^{4} \theta\left(\phi^{*} e^{2 V} \phi+\tilde{\phi}^{*} e^{-2 V} \tilde{\phi}\right),
\end{aligned}
$$

причем

$$
\frac{1}{e^{2}} Z_{3}\left(e, \frac{\Lambda}{\mu}\right)=\frac{1}{e_{0}^{2}}
$$

где $e_{0}$ - затравочная константа связи. Уравнение (20) определяет перенормированную константу связи $e$ как функцию $e_{0}$ и $\Lambda / \mu$ :

$$
e=e\left(e_{0}, \frac{\Lambda}{\mu}\right)
$$


Построив выражение для $S_{\mathrm{ren}}$, можно найти выражения для $\beta$-функции и аномальной размерности, которые в наших обозначениях определяются как

$$
\beta=\frac{d}{d \ln \mu}\left(\frac{e^{2}}{4 \pi}\right), \quad \gamma=\frac{d \ln Z(e, \Lambda / \mu)}{d \ln \mu}
$$

причем перенормированная константа связи должна быть выражена через $e_{0}$ и $\Lambda / \mu$ в соответствии с формулой (21), а значение $e_{0}$ считается фиксированным ${ }^{1)}$.

Производящий функционал для связных функций Грина в наших обозначениях записывается в виде

$$
W=-i \ln Z
$$

а эффективное действие получается с помошью преобразования Лежандра:

$$
\Gamma=W-\int d^{4} x d^{4} \theta J V-\int d^{4} x d^{2} \theta(j \phi+\tilde{j} \tilde{\phi})-\int d^{4} x d^{2} \bar{\theta}\left(j^{*} \phi^{*}+\tilde{j}^{*} \tilde{\phi}^{*}\right)
$$

причем источники $J, j$ и $\tilde{j}$ должны быть выражены через поля $V, \phi$ и $\tilde{\phi}$ при помощи уравнений

$$
V=\frac{\delta W}{\delta J}, \quad \phi=\frac{\delta W}{\delta j}, \quad \tilde{\phi}=\frac{\delta W}{\delta \tilde{j}}
$$

Заметим, что в данной работе основное внимание уделяется $\beta$-функции, определенной формулой (22). Дело в том, что в рассматриваемой теории аксиальный ток $j_{5}^{\mu}$, суперток $S^{\mu}$ и тензор энергии-импульса $\Theta^{\mu \nu}$ являются компонентами одного вешественного векторного суперполя:

$$
J^{\mu}=j_{5}^{\mu}+\bar{\theta} S^{\mu}+\bar{\theta} \gamma_{\nu} \gamma_{5} \theta \Theta^{\mu \nu}+\cdots
$$

которое в терминах суперполей $V, W_{a}, \phi$ и $\tilde{\phi}$ может быть записано в виде

$$
\begin{aligned}
J^{\mu}= & \frac{1}{e_{0}^{2}} W^{*} \gamma^{\mu} W-\frac{1}{12} Z\left(3 \bar{D}_{a}\left(\phi^{*} e^{2 V}\right) e^{-2 V}\left(\gamma^{\mu}\left(1+\gamma_{5}\right) D\right)_{a}\left(e^{2 V} \phi\right)+\right. \\
& +3 \bar{D}_{a}\left(\tilde{\phi}^{*} e^{-2 V}\right) e^{2 V}\left(\gamma^{\mu}\left(1+\gamma_{5}\right) D\right)_{a}\left(e^{-2 V} \tilde{\phi}\right)- \\
& \left.-2 \bar{D} \gamma^{\mu} \gamma_{5} D\left(\phi^{*} e^{2 V} \phi+\tilde{\phi}^{*} e^{-2 V} \tilde{\phi}\right)\right)
\end{aligned}
$$

Формулы (26) и (27) определяют выражения для $j_{5}^{\mu}, S^{\mu}$ и $\Theta^{\mu \nu}$, которые в явном виде могут быть получены с использованием формул (5), (7) и (8).

\footnotetext{
1) Эти же определения ренормгрупповых функций остаются в силе и при использовании регуляризации с помощью метода размерной редукции. При этом мы будем считать, что в $n$-мерном пространстве $e \rightarrow \mu_{0}^{2-n / 2} e$, где единица массы $\mu_{0}$ есть некоторый размерный параметр, который в наших обозначениях не полагается равным точке нормировки $\mu$.
} 
Используя выражение для дивергенции аксиального тока, несложно проверить, что на квантовом уровне закон сохранения тока $J^{\mu}$ должен выглядеть следующим образом:

$$
\left\langle\left(1+\gamma_{5}\right) \gamma^{\mu} D_{c} J_{\mu}\right\rangle=\frac{1}{8 \pi^{2}}\left(1+\gamma_{5}\right) D_{c}\left(W_{a} C^{a b} W_{b}\right)
$$

При этом угловые скобки обозначают среднее, которое соответствует функционалу (15)

$$
\begin{aligned}
\langle A\rangle \equiv & \frac{1}{\left.Z[j, J]\right|_{j=0}} \int D V D \phi D \tilde{\phi} A[\phi, \tilde{\phi}, V] \prod_{i}\left(\operatorname{det} P V\left(V, M_{i}\right)\right)^{c_{i}} \times \\
& \times \exp \left\{i \left[\frac{1}{4 e^{2}} \int d^{4} x d^{4} \theta V \partial^{2}\left(1+\frac{\partial^{2 n}}{\Lambda^{2 n}}\right) V-\right.\right. \\
& -\frac{1}{4 e^{2}}\left(Z_{3}-1\right) \int d^{4} x d^{4} \theta V \Pi_{1 / 2} \partial^{2}\left(1+\frac{\partial^{2 n}}{\Lambda^{2 n}}\right) V+ \\
& \left.\left.+\frac{1}{4} Z \int d^{4} x d^{4} \theta\left(\phi^{*} e^{2 V} \phi+\tilde{\phi}^{*} e^{-2 V} \tilde{\phi}\right)+\int d^{4} x d^{4} \theta J V\right]\right\},
\end{aligned}
$$

где источник $J$ необходимо выразить через поля с помощью (25) (при использовании других регуляризаций обозначения аналогичны).

Однако, с другой стороны, можно проверить, что аномалия следа тензора энергии-импульса $\Theta_{\mu \nu}$ должна быть пропорциональна $\beta$-функции $(22)$, что приводит к равенству

$$
\left\langle\left(1+\gamma_{5}\right) \gamma^{\mu} D_{c} J_{\mu}\right\rangle=\frac{\beta\left(\alpha_{0}\right)}{\alpha_{0}^{2}} \frac{1}{8 \pi}\left(1+\gamma_{5}\right) D_{c}\left(W_{a} C^{a b} W_{b}\right) .
$$

Поэтому суперсимметричная инвариантность требует, чтобы такая $\beta$-функция полностью определялась однопетлевым приближением:

$$
\beta(\alpha)=\frac{\alpha^{2}}{\pi}
$$

Если это по тем или иным причинам не имеет места (например, при использовании размерной редукции), то возникает противоречие, которое в литературе получило название "проблема аномалий". А поскольку в данной работе основное внимание уделяется исследованию проблемы аномалий, то наиболее удобно определять $\beta$-функцию формулой (22).

Обратим внимание, что такая $\beta$-функция, вообще говоря, отличается от функции Гелл-Манна-Лоу, которая в рассматриваемой модели может быть определена следующим образом. Если в импульсном представлении поперечную часть связной двухточечной функции Грина калибровочного поля записать в виде

$$
\begin{gathered}
\left.\Pi_{1 / 2} \frac{1}{2} \int d^{4} x d^{4} y \frac{\delta^{2} W}{\delta J_{x} \delta J_{y}}\right|_{J=0} \exp \left(i p_{\mu} x^{\mu}+i q_{\mu} y^{\mu}\right)= \\
=(2 \pi)^{4} \delta^{4}(p+q) \Pi_{1 / 2} \delta^{4}\left(\theta_{x}-\theta_{y}\right) d\left(\alpha, \frac{p}{\mu}\right)
\end{gathered}
$$


где

$$
\Pi_{1 / 2}=-\frac{1}{16 \partial^{2}} D^{a} \bar{D}^{2} C_{a b} D^{b}
$$

- суперсимметричный поперечный проектор, то функцию Гелл-Манна-Лоу по определению полагаем равной

$$
\left.\tilde{\beta}(\tilde{\alpha}) \equiv \frac{\partial}{\partial x}\left(\frac{d(\alpha, x)}{4 \pi}\right)\right|_{x=1},
$$

где $\tilde{\alpha}=d(\alpha, 1)$. При этом известно [34], что функция Гелл-Манна-Лоу связана с определенной выше $\beta$-функцией следуюшим соотношением:

$$
\tilde{\beta}(\tilde{\alpha})=\beta(\alpha) \frac{d \tilde{\alpha}}{d \alpha} .
$$

Поэтому отличие $\beta$-функции (22), рассматриваемой как функция $\alpha$, от $\beta$-функции (34) фактически связано с возможностью переопределения перенормированной константы связи [35]. Тем не менее из формулы (35) несложно видеть, что в разложениях функций $\beta$ и $\tilde{\beta}$ в ряд по $\alpha$ первые два коэффициента будут одинаковыми.

Важно обратить внимание на то, что при выводе формулы (35) всегда подразумевается отсутствие зависимости производяшего функционала $\left.Z[j, J]\right|_{j=0}$ от точки нормировки $\mu$, если значение голой константы связи $e_{0}$ предполагается фиксированным и не зависяшим от точки нормировки $\mu$. Ниже мы увидим, что в силу сушествования аномалии масштабирования для $N=1$ суперсимметричной электродинамики в голоморфной нормировке (в которой справедлива теорема Адлера-Бардина) это уже не так. Поэтому формула (35) не будет верна в этом случае. Возможно, что зависимость производяшего функционала от точки нормировки указывает на некорректность использования голоморфной нормировки для суперсимметричных теорий, однако этот вопрос здесь не обсуждается. Цель данной работы состоит только в том, чтобы показать отсутствие противоречия между теоремой Адлера-Бардина и структурой квантовых поправок в высших петлях.

2.3. Размерная редукция и размерная регуляризация. Несмотря на то что метод высших ковариантных производных может быть без каких-либо особенных сложностей применен к суперсимметричной электродинамике [25], [26], [36], в неабелевых теориях его использование связано со значительными техническими проблемами, возникаюшими из-за сложной структуры вершин взаимодействия. Поэтому при проведении конкретных вычислений в теории поля этот метод применялся значительно реже, чем размерная регуляризация [29].

В размерной регуляризации все вычисления формально проводятся в пространстве некоторой (не обязательно целой) размерности $n$. Однако в применении к суперсимметричным теориям такая регуляризация оказывается очень неудобной, поскольку ее введение нарушает суперсимметрию. В частности, равенство чисел бозонных и фермионных степеней свободы может иметь место только при определенных значениях размерности $n$. 
Для того чтобы построить простой метод вычислений для суперсимметричных теорий, в работе [27] была построена регуляризация при помоши метода размерной редукции. В соответствии с этим методом функция Лагранжа в четырех измерениях размерно редуцируется к некоторой размерности $n<4$. При этом, например, четырехмерное векторное поле $A_{\mu}$ распадается на $n$-компонентный вектор $\tilde{A}_{\mu}$ и $\epsilon=4-n$ скалярных полей $\hat{A}_{\mu}$, тогда как полное число степеней свободы остается таким же, как и в четырехмерном случае. Очень важно, что при этом размерность $n$ с необходимостью выбирается меньше 4. В частности, из этого следует, что

$$
\delta_{\mu}^{\nu} \tilde{\delta}_{\nu}^{\mu}=n
$$

где $\delta_{\mu}^{\nu}$ - символ Кронекера в четырех измерениях, а $\tilde{\delta}_{\mu}^{\nu}$ - символ Кронекера в $n$ измерениях.

Однако, как было отмечено в работе [28], такой метод является внутренне противоречивым, поскольку, например, произведение $\tilde{\varepsilon}^{\alpha \beta \gamma \delta} \tilde{\varepsilon}_{\alpha \beta \gamma \delta} \hat{\varepsilon}^{\mu \nu \rho \tau} \hat{\varepsilon}_{\mu \nu \rho \tau}$ в зависимости от способа вычисления оказывается равным либо нулю, либо $(n-4)(n-3)^{2}(n-2)^{2} \times$ $(n-1)^{2} n$, благодаря чему метод размерной редукции лишен внутренних противоречий только при $n=0,1,2,3,4$. Кроме того, из-за требования $n<4$ матрица $\gamma_{5}$ должна одновременно удовлетворять условиям

$$
\left\{\gamma_{5}, \tilde{\gamma}_{\mu}\right\}=0, \quad \gamma_{5}^{2}=1
$$

В качестве следствия можно получить (см. [37]), что

$$
(n-4) \operatorname{tr}\left(\gamma_{5} \tilde{\gamma}_{\mu} \tilde{\gamma}_{\nu} \tilde{\gamma}_{\alpha} \tilde{\gamma}_{\beta}\right)=0
$$

В силу условий (37) и калибровочная, и киральная симметрии оказываются ненарушенными. В размерной регуляризации аналогичная проблема обходится за счет того, что при $n>4$

$$
\left\{\gamma_{5}, \gamma_{\mu}\right\}=0, \quad \mu=0, \ldots, 3 ; \quad\left[\gamma_{5}, \gamma_{\mu}\right]=0, \quad \mu>3
$$

В частности, именно эти свойства позволяют получить в методе размерной регуляризации правильное значение аксиальной аномалии [29]. Тем не менее метод размерной редукции считается пригодным для петлевых вычислений в суперсимметричных теориях (см., например, обзор [37] и ссылки в нем). При этом с практической точки зрения он сводится к использованию четырехмерной алгебры $\gamma$-матриц и вычислению оставшихся интегралов при $n \neq 4[32]$. 


\section{3. ВЫЧИСЛЕНИЕ ДВУХПЕТЛЕВОЙ $\beta$-ФУНКЦИИ В РАЗЛИЧНЫХ РЕГУЛЯРИЗАЦИЯХ}

3.1. Двухпетлевые диаграммы. Диаграммы Фейнмана, дающие нетривиальный вклад в двухпетлевую $\beta$-функцию $N=1$ суперсимметричной электродинамики, показаны на рисунке. Эти диаграммы могут быть естественным образом разделены на три части:

1) однопетлевые диаграммы а, б;

2) двухпетлевые диаграммы без контрчленных вставок в-з;

3) диаграммы со вставками однопетлевых контрчленов и-м.

3.2. Регуляризация высшими производными. При использовании регуляризации методом высших производных для суммы диаграмм каждого типа получаются следующие результаты $[25],[36]^{2)}$ :

$$
\Delta \Gamma_{V}^{(2)}=\operatorname{Re} \int d^{2} \theta \frac{d^{4} p}{(2 \pi)^{4}} W_{a}(p) C^{a b} W_{b}(-p)\left(f_{1}+f_{2}+f_{2 \mathrm{PV}}+f_{3}\right),
$$

где

$$
f_{1}=-\frac{i}{2}\left(\int \frac{d^{4} k}{(2 \pi)^{4}} \frac{1}{k^{2}(k+p)^{2}}-\sum_{i} c_{i} \int \frac{d^{4} k}{(2 \pi)^{4}} \frac{1}{\left(k^{2}-M_{i}^{2}\right)\left((k+p)^{2}-M_{i}^{2}\right)}\right)
$$

представляет собой однопетлевой результат с учетом вклада полей Паули-Вилларса,

$$
f_{2}=-e^{2} \int \frac{d^{4} k}{(2 \pi)^{4}} \frac{d^{4} q}{(2 \pi)^{4}} \frac{(k+p+q)^{2}+q^{2}-k^{2}-p^{2}}{k^{2}\left(1+(-1)^{n} k^{2 n} / \Lambda^{2 n}\right)(k+q)^{2}(k+p+q)^{2} q^{2}(q+p)^{2}}
$$

есть сумма диаграмм в-3 без учета вкладов полей Паули-Вилларса,

$$
\begin{aligned}
f_{2 \mathrm{PV}}= & e^{2} \sum_{i} c_{i} \int \frac{d^{4} k}{(2 \pi)^{4}} \frac{d^{4} q}{(2 \pi)^{4}} \frac{1}{k^{2}\left(1+(-1)^{n} k^{2 n} / \Lambda^{2 n}\right)} \times \\
& \times\left[\frac{(k+p+q)^{2}+q^{2}-k^{2}-p^{2}}{\left((k+q)^{2}-M_{i}^{2}\right)\left((k+p+q)^{2}-M_{i}^{2}\right)\left(q^{2}-M_{i}^{2}\right)\left((q+p)^{2}-M_{i}^{2}\right)}+\right. \\
& \left.+\frac{4 M_{i}^{2}}{\left((k+q)^{2}-M_{i}^{2}\right)\left(q^{2}-M_{i}^{2}\right)^{2}\left((q+p)^{2}-M_{i}^{2}\right)}\right]
\end{aligned}
$$

есть вклад диаграмм в-3 с внутренней петлей полей Паули-Вилларса и, наконец,

$$
f_{3}=-\frac{i e^{2}}{2 \pi^{2}} \ln \frac{\Lambda}{\mu} \sum_{i} c_{i} \int \frac{d^{4} k}{(2 \pi)^{4}} \frac{M_{i}^{2}}{\left(k^{2}-M_{i}^{2}\right)^{2}\left((k+p)^{2}-M_{i}^{2}\right)}
$$

есть вклад диаграмм со вставкой однопетлевых контрчленов и-м.

\footnotetext{
2) В работе [36] правильно вычислены вклады диаграмм а-3, но пропущен вклад от суммы диаграмм и-м.
} 

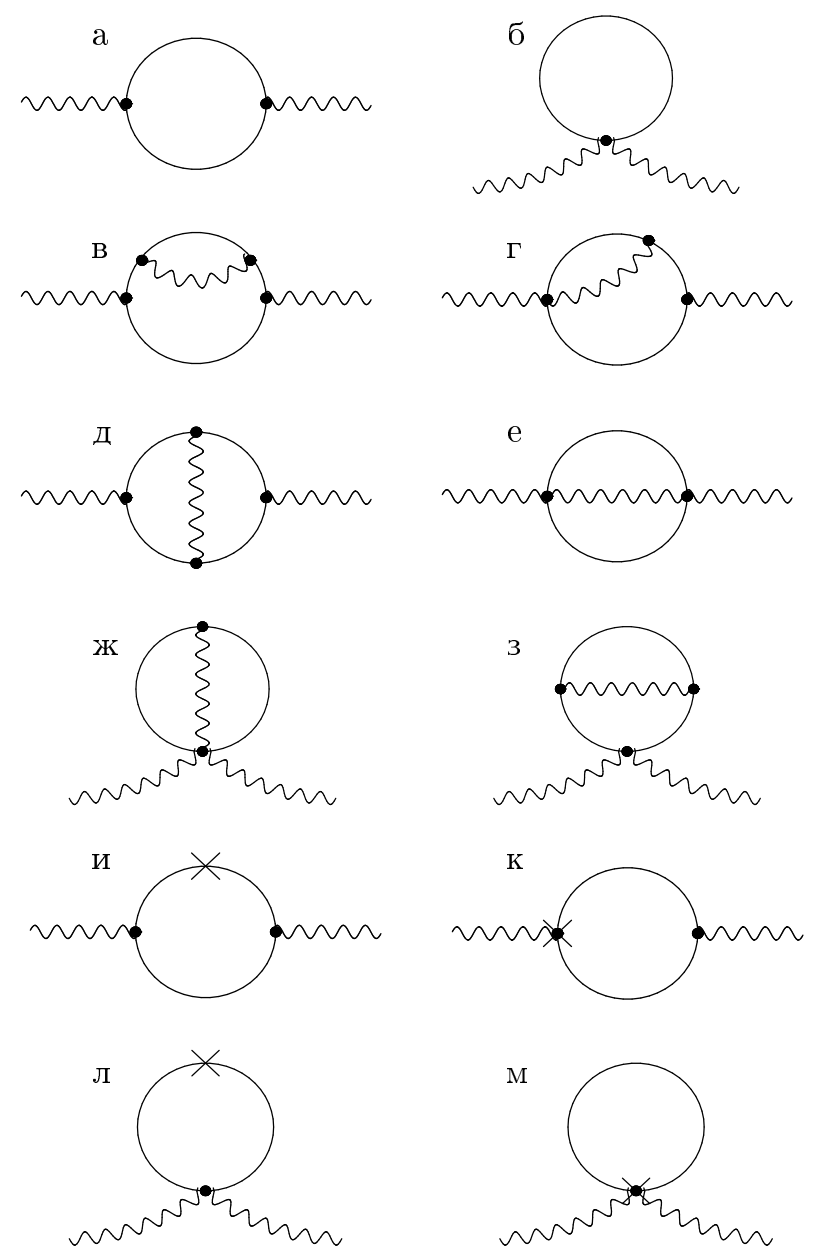

Вычисления, проведенные в работах [25], [36], показывают, что вклад (43) является конечным как в ультрафиолетовой, так и в инфракрасной области и не влияет на расходяшуюся часть эффективного действия. Это утверждение является крайне важным, поскольку ряд диаграмм с полями Паули-Вилларса содержат расходяшиеся поддиаграммы. Поэтому конечность суммы таких диаграмм означает, что нет необходимости добавлять в теорию еше какую-либо дополнительную регуляризацию для этих диаграмм (по крайней мере, на двухпетлевом уровне). Действительно, регуляризация Паули-Вилларса заведомо подразумевает сушествование расходяшихся диаграмм и сокрашение расходимостей только в их сумме. Поэтому сушествование расходимостей в отдельных диаграммах проблемой не является. 
Для остальных вкладов в формуле (40) получаются следуюшие результаты:

$$
\begin{aligned}
& f_{1}=\frac{1}{16 \pi^{2}} \ln \frac{\Lambda}{p}+O(1), \\
& f_{2}=\frac{1}{16 \pi^{2}} \frac{\alpha}{\pi} \ln \frac{\Lambda}{p}+O(1), \\
& f_{3}=-\frac{1}{16 \pi^{2}} \frac{\alpha}{\pi} \ln \frac{\Lambda}{\mu}+o(1),
\end{aligned}
$$

при этом через $O(1)$ обозначены величины, которые в пределе $\Lambda \rightarrow \infty$ дают некоторую числовую постоянную, а через $o(1)$ - члены, которые исчезают в этом пределе.

Поэтому сумма однопетлевого и двухпетлевого вкладов в эффективное действие оказывается равной $\left(\Gamma_{\mathrm{R}}=S_{\text {ren }}+\Delta \Gamma\right)$

$$
\Delta \Gamma_{V}^{(2)}=\frac{1}{16 \pi^{2}} \operatorname{Re} \int d^{2} \theta \frac{d^{4} p}{(2 \pi)^{4}} W_{a}(p) C^{a b} W_{b}(-p)\left(\ln \frac{\Lambda}{p}+\frac{\alpha}{\pi} \ln \frac{\mu}{p}+O(1)\right)
$$

Из этой формулы видно, что для компенсации двухпетлевых расходимостей не нужно добавлять никаких контрчленов. Поэтому при использовании регуляризации высшими производными в двухпетлевом приближении контрчлены могут быть записаны в виде

$$
\begin{aligned}
\Delta S^{(2)}= & -\frac{1}{16 \pi^{2}} \operatorname{Re} \int d^{2} \theta \frac{d^{4} p}{(2 \pi)^{4}} W_{a}(p) C^{a b} W_{b}(-p) \times \\
& \times\left(\ln \frac{\Lambda}{\mu}+\text { конечная постоянная }+O\left(\alpha^{2}\right)\right)+ \\
& + \text { слагаемые с суперполями материи, }
\end{aligned}
$$

откуда следует, что

$$
\frac{1}{\alpha_{0}}=\frac{1}{\alpha}-\frac{1}{\pi} \ln \frac{\Lambda}{\mu}+O\left(\alpha^{2}\right) .
$$

В силу формулы (22) это выражение соответствует следуюшей двухпетлевой $\beta$-функции:

$$
\beta_{\mathrm{hd}}=\frac{\alpha^{2}}{\pi}+O\left(\alpha^{4}\right)
$$

Из формул (46) и (47) также следует, что перенормированное эффективное действие с точностью до конечных постоянных записывается в виде

$$
\begin{aligned}
\left(\Gamma_{V}^{(2)}\right)_{\mathrm{R}} & =S+\Delta S^{(2)}+\Delta \Gamma_{V}^{(2)}= \\
& =\frac{1}{16 \pi^{2}} \operatorname{Re} \int d^{2} \theta \frac{d^{4} p}{(2 \pi)^{4}} W_{a}(p) C^{a b} W_{b}(-p)\left(\frac{\pi}{\alpha}+\ln \frac{\mu}{p}+\frac{\alpha}{\pi} \ln \frac{\mu}{p}+O(1)\right) .
\end{aligned}
$$

3 Теоретическая и математическая физика, т. 140, № 1, 2004 г. 
Отсюда, в частности, получаем

$$
d\left(\frac{p}{\mu}\right)=\alpha\left(1-\frac{\alpha}{\pi} \ln \frac{p}{\mu}+\alpha c_{1}-\frac{\alpha^{2}}{\pi^{2}} \ln \frac{p}{\mu}+\alpha^{2} c_{2}+O\left(\alpha^{3}\right)\right)^{-1}
$$

где $c_{1}$ и $c_{2}$ - некоторые конечные постоянные. Поэтому несложно убедиться, что функция $\tilde{\beta}$, определенная формулой $(34)$, оказывается равной

$$
\tilde{\beta}(\alpha)=\frac{\alpha^{2}}{\pi^{2}}\left(1+\frac{\alpha}{\pi}+O\left(\alpha^{2}\right)\right) .
$$

3.3. Размерная редукция. Результат, соответствующий формуле (40) при использовании регуляризации методом размерной редукции, записывается в виде

$$
\Delta \Gamma_{V}^{(2)}=\operatorname{Re} \int d^{2} \theta \frac{d^{4} p}{(2 \pi)^{4}} W_{a}(p) C^{a b} W_{b}(-p)\left(\tilde{f}_{1}+\tilde{f}_{2}+\tilde{f}_{3}\right)
$$

где

$$
\begin{aligned}
& \tilde{f}_{1}=-\frac{i}{2} \int \frac{d^{n} k}{(2 \pi)^{4}} \frac{1}{k^{2}(k+p)^{2}}, \\
& \tilde{f}_{2}=-e^{2} \int \frac{d^{n} k}{(2 \pi)^{4}} \frac{d^{n} q}{(2 \pi)^{4}} \frac{(k+p+q)^{2}+q^{2}-k^{2}-p^{2}}{k^{2}(k+q)^{2}(k+p+q)^{2} q^{2}(q+p)^{2}}, \\
& \tilde{f}_{3}=0 .
\end{aligned}
$$

При использовании обозначений, заимствованных из работы [31],

$$
\begin{aligned}
I & \equiv\left(p^{2}\right)^{2-n / 2} \int \frac{d^{n} k}{k^{2}(k+p)^{2}}=\pi^{n / 2} \Gamma\left(2-\frac{n}{2}\right) B\left(\frac{n}{2}-1, \frac{n}{2}-1\right) \\
J & \equiv\left(p^{2}\right)^{4-n} \int \frac{d^{n} k}{\left(k^{2}\right)^{3-n / 2}(k+p)^{2}}=\pi^{n / 2} \frac{\Gamma(4-n)}{\Gamma(3-n / 2)} B\left(n-3, \frac{n}{2}-1\right) \\
Z & \equiv\left(p^{2}\right)^{5-n} \int \frac{d^{n} k d^{n} q}{q^{2} k^{2}(k+p)^{2}(q+p)^{2}(k-q)^{2}}=\frac{1}{n-4}\left((6 n-20) I J-(2 n-6) I^{2}\right),
\end{aligned}
$$

двухпетлевой вклад в эффективное действие в случае регуляризации методом размерной редукции может быть записан в виде

$$
\begin{aligned}
\Delta \Gamma_{V}^{(2)}= & \operatorname{Re} \int d^{2} \theta \frac{d^{4} p}{(2 \pi)^{4}} W_{a}(p) C^{a b} W_{b}(-p) \times \\
& \times\left(\frac{1}{2(2 \pi)^{4}}\left(\frac{p}{\mu_{0}}\right)^{n-4} I+\frac{e^{2}}{(2 \pi)^{8}}\left(\frac{p}{\mu_{0}}\right)^{2 n-8}\left(2 I J-I^{2}-p^{2} Z\right)\right),
\end{aligned}
$$

где параметр $\mu_{0}$ - так называемая единица массы [38] - учитывает зависимость размерности константы связи от размерности пространства $n$. 
После вычисления интегралов $I, J, Z$ получаем, что

$$
\begin{aligned}
\Delta \Gamma_{V}^{(2)}= & \frac{1}{16 \pi^{2}} \operatorname{Re} \int d^{2} \theta \frac{d^{4} p}{(2 \pi)^{4}} W_{a}(p) C^{a b} W_{b}(-p) \times \\
& \times\left(\frac{1}{4-n}+\ln \frac{\mu_{0}}{p}+\frac{\alpha}{\pi}\left(\frac{1}{2(4-n)}+\ln \frac{\mu_{0}}{p}\right)+O(1)\right) .
\end{aligned}
$$

Первые два слагаемых в этой формуле соответствуют однопетлевому интегралу $\tilde{f}_{1}$, а третье - двухпетлевому интегралу $\tilde{f}_{2}$.

Для устранения расходимостей в формуле (57) необходимо добавить контрчлены

$$
\begin{aligned}
\Delta S^{(2)}= & \frac{1}{16 \pi^{2}} \operatorname{Re} \int d^{2} \theta \frac{d^{4} p}{(2 \pi)^{4}} W_{a}(p) C^{a b} W_{b}(-p)\left(-\frac{1}{4-n}+\ln \frac{\mu}{\mu_{0}}+\right. \\
& \left.+\frac{\alpha}{\pi}\left(-\frac{1}{2(4-n)}+\ln \frac{\mu}{\mu_{0}}\right)+\text { конечные постоянные }+O\left(\alpha^{2}\right)\right),
\end{aligned}
$$

откуда следует, что в методе размерной редукции $\beta$-функция, определенная формулой (22), оказывается равной

$$
\beta_{\text {dred }}=\frac{\alpha^{2}}{\pi}+\frac{\alpha^{3}}{\pi^{2}}+O\left(\alpha^{4}\right) .
$$

В отличие от чисто однопетлевого результата (49) формула (59) согласуется с предсказанием, следующим из точной $\beta$-функции НШВЗ

$$
\beta_{\text {ншв3 }}=\frac{\alpha^{2}}{\pi}(1-\gamma(\alpha)),
$$

где $\gamma$ - аномальная размерность, определяемая формулой (22).

Несложно видеть, что из формул (57) и (58) следует, что перенормированное эффективное действие

$$
\left(\Gamma_{V}^{(2)}\right)_{\mathrm{R}}=S+\Delta S^{(2)}+\Delta \Gamma_{V}^{(2)}
$$

так же как и в случае регуляризации высшими производными, будет даваться формулой (50). Следовательно, в двухпетлевом приближении для обеих регуляризаций совпадут и функции Гелл-Манна-Лоу (34).

3.4. Сравнение регуляризаций высшими производными и размерной редукцией. Сравнение описанных выше вычислений при помощи метода высших производных и при помощи метода размерной редукции показывает, что различие между результатами полностью обусловлено различием в выражениях для суммы диаграмм с контрчленными вставками. Для того чтобы понять причину этого различия, необходимо заметить, что в двухпетлевом приближении сумма диаграмм с контрчленными вставками пропорциональна однопетлевому вкладу в аномалию Кониши [14], [15]

$$
\left\langle\frac{1}{4} Z \int d^{4} x d^{4} \theta\left(\phi^{*} e^{2 V} \phi+\tilde{\phi}^{*} e^{-2 V} \tilde{\phi}\right)\right\rangle=-\frac{1}{16 \pi^{2}} \operatorname{Re} \int d^{4} x d^{2} \theta W_{a} C^{a b} W_{b} .
$$


Это утверждение легко проверить явно, вьполняя разложение производящего функционала с помощью метода перевала.

Аномалия Кониши тесно связана с аксиальной аномалией. Напомним кратко, откуда происходит такая связь. Рассмотрим вначале следующее выражение:

$$
\operatorname{Im}\left[Z \bar{D}^{2}\left(\phi^{*} e^{2 V} \phi+\tilde{\phi}^{*} e^{-2 V} \tilde{\phi}\right)\right]
$$

Используя разложения (5) и (7), несложно убедиться, что при записи в терминах компонентных полей оно будет содержать (наряду с другими членами) выражение

$$
-\bar{\theta} \theta Z \partial_{\mu}\left(\bar{\Psi} \gamma^{\mu} \gamma_{5} \Psi\right)
$$

где дираковский спинор $\Psi$ определяется формулой (6). Хорошо известно [39], что закон сохранения входящего в это выражение аксиального тока нарушается квантовыми поправками. В частности, вакуумное среднее от выражения (64) оказывается равным

$$
-\bar{\theta} \theta \frac{1}{8 \pi^{2}} F_{\mu \nu} \widetilde{F}^{\mu \nu}
$$

В силу суперсимметричной инвариантности выражения (63) его вакуумное среднее должно быть суперполем и в качестве одной из составляющих должно содержать выражение (65). Эти требования приводят к равенству 3 )

$$
\operatorname{Im}\left\langle Z \bar{D}^{2}\left(\phi^{*} e^{2 V} \phi+\tilde{\phi}^{*} e^{-2 V} \tilde{\phi}\right)\right\rangle=\frac{1}{2 \pi^{2}} \operatorname{Im}\left(W_{a} C^{a b} W_{b}\right)
$$

Используя законы изменения компонентных полей при преобразованиях суперсимметрии, несложно убедиться, что если мнимая часть некоторого кирального суперполя равна нулю, то все это суперполе равно произвольной вешественной постоянной. Поэтому из формулы (66) следует, что

$$
\left\langle Z \bar{D}^{2}\left(\phi^{*} e^{2 V} \phi+\tilde{\phi}^{*} e^{-2 V} \tilde{\phi}\right)\right\rangle=\frac{1}{2 \pi^{2}} W_{a} C^{a b} W_{b}+\text { const }
$$

В принципе можно доказать, что вещественная постоянная в этом равенстве равна нулю, однако для наших целей вполне приемлема формула (67). Применяя к обеим ее частям оператор

$$
-\frac{1}{2} \int d^{4} x D^{2}=\int d^{4} x d^{2} \theta
$$

3) Заметим, что приведенные рассуждения, строго говоря, доказательством не являются, поскольку формула (64) не содержит всех членов разложения выражения (63), пропорциональных $\bar{\theta} \theta$. Строгий вывод аномалии Кониши дан в работах [14], [15]. В принципе его можно сформулировать и исходя из результатов вычисления методом высших производных, приведенных в данной работе. Целью этих рассуждений было именно пояснение связи аксиальной аномалии и аномалии Кониши. 
и выгисляя вешественную часть, получаем, что суперсимметрия и нетривиальное значение аксиальной аномалии приводят к тождеству (62).

Очень важно заметить, что если по каким-либо причинам треугольные диаграммы, определяющие аксиальную аномалию, оказались равными нулю, а суперсимметрия при вычислениях нигде не нарушалась, то вместо тождества (62) будет автоматически получаться равенство

$$
\left\langle\frac{1}{4} Z \int d^{4} x d^{4} \theta\left(\phi^{*} e^{2 V} \phi+\tilde{\phi}^{*} e^{-2 V} \tilde{\phi}\right)\right\rangle=0
$$

Именно такая ситуация и имеет место при использовании метода размерной редукции. Действительно, если мы работаем в формализме суперграфов, то дополнительные условия типа $\operatorname{tr}(A B) \neq \operatorname{tr}(B A)$, которые обычно накладываются при вычислении аксиальной аномалии, автоматически не удовлетворяются. В результате вычисления с необходимостью проводятся с использованием исходного варианта метода, предложенного в работе [27]. Но в рамках этого метода размерность пространства обязательно должна считаться меньше 4. Поэтому (в отличие от размерной регуляризации) матрица $\gamma_{5}$ в размерной редукции антикоммутирует со всеми $\gamma$-матрицами, благодаря чему киральная симметрия в регуляризованной теории сохраняется. Однако полученное противоречие просто является следствием описанных вьше внутренних противоречий метода регуляризации при помощи размерной редукции.

Итак, противоречивость метода размерной редукции в отсутствие дополнительных предположений ведет к нулевому вкладу диаграмм с контрчленными вставками, что полностью подтверждается описанными выше явными вычислениями. А поскольку вклад этих диаграмм в двухпетлевом приближении должен компенсировать все остальные вклады, то размерная редукция дает нетривиальную двухпетлевую $\beta$-функцию (22) и приводит к проблеме аномалий.

\section{4. ТОЧНЫЙ РЕЗУЛЬТАТ ДЛЯ СУММЫ ДИАГРАММ С КОНТРЧЛЕННЫМИ ВСТАВКАМИ}

Выше было показано, что разница результатов при вычислениях с использованием регуляризаций высшими производными и размерной редукцией определяется суммой диаграмм с контрчленными вставками, которая при использовании размерной редукции заведомо будет равна нулю в силу неприспособленности метода размерной редукции к вычислению аномалии Кониши. Для того чтобы дать еше более убедительное подтверждение правильности результатов, изложенных выше, можно попробовать вычислить сумму диаграмм с контрчленными вставками точно во всех петлях с использованием метода высших производных. 
Применяя формализм функционального интеграла, соответствующий результат, полученный в приложении, можно сформулировать следующим образом:

$$
\begin{aligned}
e^{\left.i \Gamma\right|_{\phi=0}=} & \exp \left(-i \ln Z \frac{1}{16 \pi^{2}} \operatorname{Re} \int d^{4} x d^{2} \theta W_{a} C^{a b} W_{b}+\text { конечные члены }\right) \times \\
& \times \int D V D \phi D \tilde{\phi} \prod_{i}\left(\operatorname{det}^{\prime} P V\left(V, M_{i}\right)\right)^{c_{i}} \times \\
& \times \exp \left\{i \left[\frac{1}{4 e^{2}} \int d^{4} x d^{4} \theta V \partial^{2}\left(1+\frac{\partial^{2 n}}{\Lambda^{2 n}}\right) V-\right.\right. \\
& -\frac{1}{4 e^{2}}\left(Z_{3}-1\right) \int d^{4} x d^{4} \theta V \Pi_{1 / 2} \partial^{2}\left(1+\frac{\partial^{2 n}}{\Lambda^{2 n}}\right) V+ \\
& \left.\left.+\frac{1}{4} \int d^{4} x d^{4} \theta\left(\phi^{*} e^{2 V} \phi+\tilde{\phi}^{*} e^{-2 V} \tilde{\phi}\right)+\int d^{4} x d^{4} \theta J V\right]\right\},
\end{aligned}
$$

причем источник $J$, как обычно, должен быть выражен через поле $V$ и было использовано обозначение

$$
\begin{aligned}
\left(\operatorname{det}^{\prime} P V(V, M)\right)^{-1} \equiv & \int D \Phi D \tilde{\Phi} \exp \left\{i \left[\frac{1}{4} \int d^{4} x d^{4} \theta\left(\Phi^{*} e^{2 V} \Phi+\tilde{\Phi}^{*} e^{-2 V} \tilde{\Phi}\right)+\right.\right. \\
& \left.\left.+\frac{1}{2} \int d^{4} x d^{2} \theta M \tilde{\Phi} \Phi+\frac{1}{2} \int d^{4} x d^{2} \bar{\theta} M \tilde{\Phi}^{*} \Phi^{*}\right]\right\} .
\end{aligned}
$$

(Разница между этим определением и формулой (16) заключается в отсутствии константы $Z$ в определении $\operatorname{det}^{\prime}$.)

В принципе эту формулу формально можно записать и в более простом виде:

$$
\begin{aligned}
& \left\langle\exp \left(i(Z-1) \frac{1}{4} \int d^{4} x d^{4} \theta\left(\phi^{*} e^{2 V} \phi+\tilde{\phi}^{*} e^{-2 V} \tilde{\phi}\right)\right)\right\rangle= \\
& =\exp \left(-i \ln Z \frac{1}{16 \pi^{2}} \operatorname{Re} \int d^{4} x d^{2} \theta W_{a} C^{a b} W_{b}+\text { конечные члены }\right) .
\end{aligned}
$$

Поэтому, несмотря на достаточно сложную структуру формулы (69), ее смысл достаточно простой: после одновременного масштабирования полей $\phi, \Phi, \tilde{\phi}$ и $\tilde{\Phi}$ в производящем функционале $\left.Z[j, J]\right|_{j=0}$ появляется дополнительный множитель

$$
\exp \left(-i \ln Z \frac{1}{16 \pi^{2}} \operatorname{Re} \int d^{4} x d^{2} \theta W_{a} C^{a b} W_{b}+\text { конечные члены }\right)
$$

Важно заметить, что существование такого аномального вклада при использовании голоморфной нормировки суперполей материи не позволяет удалить зависимость производяшего функционала при $j=0$ от точки нормировки с помошью масштабирования 
$\phi \rightarrow Z^{-1 / 2} \phi$, поскольку $\ln Z$ в аномальном вкладе будет зависеть от $\mu$ (при фиксированном значении $\left.e_{0}\right)$. Явно увидеть зависимость, например, эффективного действия от $\mu$ в этом случае можно, если сравнить формулы (48) и (50). Возможно, что это указывает на некорректность использования голоморфной нормировки при вычислениях в суперсимметричных теориях, но мы все же будем рассматривать этот случай, поскольку, как несложно доказать, теорема Адлера-Бардина справедлива именно в такой нормировке. В силу зависимости производяшего функционала от точки нормировки функции $\beta$ и $\tilde{\beta}$, определенные ранее, окажутся различными даже в двухпетлевом приближении. При этом $\beta$-функция, определенная формулой (22), будет однопетлевой, как это и требует структура супермультиплета аномалий, тогда как $\beta$-функция, определенная формулой (34) будет совпадать с точной $\beta$-функцией НШВЗ (возможно, с точностью до переопределения перенормированной константы связи).

Действительно, из формулы (69) следует, что сумма контрчленных диаграмм дает вклад в $\beta$-функцию

$$
\Delta \beta=\frac{\alpha^{2}}{\pi} \frac{d \ln Z}{d \ln \mu}=\frac{\alpha^{2}}{\pi} \gamma(\alpha) .
$$

Поэтому если при использовании регуляризации с помошью высших производных получается, что $\beta$-функция является чисто однопетлевой, то сумма всех диаграмм без контрчленных вставок в методе высших производных будет соответствовать точной $\beta$-функции НШВЗ (60). Такой результат можно получить, например, проводя в перенормированном действии перемасштабирование суперполей материи $\phi \rightarrow Z^{-1 / 2} \phi$, которое переводит перенормированное действие (19) в

$$
\begin{aligned}
S_{\mathrm{ren}}= & \frac{1}{4 e^{2}} Z_{3} \operatorname{Re} \int d^{4} x d^{2} \theta W_{a} C^{a b}\left(1+\frac{\partial^{2 n}}{\Lambda^{2 n}}\right) W_{b}+ \\
& +\frac{1}{4} \int d^{4} x d^{4} \theta\left(\phi^{*} e^{2 V} \phi+\tilde{\phi}^{*} e^{-2 V} \tilde{\phi}\right) .
\end{aligned}
$$

Если сразу подставить такое перенормированное действие в $Z$, то при $j=0$ производящий функционал уже не будет зависеть от $\mu$ и формула (35) будет справедлива. В этом случае диаграммы с контрчленными вставками в линиях суперполей материи возникать не будут и в двухпетлевом приближении функции $\beta$ и $\tilde{\beta}$ окажутся одинаковыми, что действительно следует из проведенных вычислений. При этом обе эти функции будут иметь поправки во всех петлях, однако для канонической нормировки (72) теорема Адлера-Бардина уже не будет иметь место.

Другая возможность получить результат (60) заключается в проведении вычислений с помощью метода размерной редукции. При этом появляется проблема аномалий, которая вызывается неприспособленностью этой регуляризации к вычислению аномалий. Однако после проведения перенормировки эффективное действие все же совпадет с эффективным действием, полученным в методе высших производных, и физические результаты будут точно такими же. 
В заключение необходимо отметить, что формулы, аналогичные тождеству (70) были также получены в работах [13] и [21]. Однако интерпретация левой части уравнения (70) как суммы диаграмм с контрчленными вставками при этом не давалась, несмотря на то что вывод этого уравнения, приведенный в статье [13], в определенной степени близок к описанному в приложении.

\section{5. ЗАКЛЮЧЕНИЕ}

В данной работе был проведен анализ вычисления двухпетлевой $\beta$-функции в $N=1$ суперсимметричной электродинамике с использованием различных методов регуляризации. Полученные результаты можно сформулировать следующим образом:

1. Аномалия Кониши действительно влияет на величину $\beta$-функции, определенную формулой (22), как это было замечено в работе [13]. С точки зрения диаграммной техники аномалия Кониши возникает как сумма диаграмм с контрчленными вставками. Тем не менее в двухпетлевом приближении перенормированное действие при учете аномалии Кониши имеет точно тот же вид, что и при ее отсутствии.

2. Возникновение аномалии масштабирования, исследованной в работе [21], с точки зрения диаграммной техники может быть легко объяснено: диаграммы с контрчленными вставками появляются только в случае, если в перенормированном действии нормировка суперполя материи выбирается в виде

$$
\frac{1}{4} Z \int d^{4} x d^{4} \theta\left(\phi^{*} e^{2 V} \phi+\tilde{\phi}^{*} e^{-2 V} \tilde{\phi}\right) .
$$

После масштабирования $\phi \rightarrow Z^{-1 / 2} \phi$ соответствуюшие слагаемые уже не будут давать диаграмм с контрчленными вставками. Поэтому благодаря аномалии Кониши возникает аномалия масштабирования.

3. При вычислении $\beta$-функции с использованием непротиворечивой регуляризации, сохраняющей суперсимметрию, проблема аномалий не возникает (по крайней мере, во второй петле) и $\beta$-функция, определенная формулой (22), оказывается чисто однопетлевой. Для рассматриваемой модели таким методом является регуляризация при помощи высших производных. Важно, что с помошью метода высших производных удается получить точное во всех порядках теории возмушений выражение для аномалии масштабирования, согласуюшееся с результатами, полученными в работах [13] и [21] из других соображений.

В высших петлях (начиная с третьей) $\beta$-функция как функция $\alpha$ является схемно-зависимой, благодаря чему наиболее интересно проверить равенство нулю трехпетлевого вклада в $\beta$-функцию при использовании метода высших производных и различных схем вычитаний. Соответствуюшая работа в настоящее время закончена [33].

4. Поскольку в двухпетлевом приближении перенормированные эффективные действия в обеих регуляризациях совпадают, то совпадают и функции Гелл-Манна-Лоу, которые имеют поправки во всех петлях. Однако структура мультиплета аномалий тре- 
бует, чтобы однопетлевой была $\beta$-функция (22), тогда как для функции $\tilde{\beta}$, определенной формулой (34), это совсем не обязательно.

Конечно, кажется, что различие коэффициентов при $\alpha^{3}$ в функциях $\beta$ и $\tilde{\beta}$ при использовании регуляризации высшими производными противоречит утверждению о том, что второй коэффипиент $\beta$-функции является схемно-независимым. Однако связь между функциями $\beta$ и $\tilde{\beta}$ в присутствии аномалии Кониши должна анализироваться очень аккуратно. Действительно, в силу наличия аномалии масштабирования при использовании голоморфной нормировки имеется зависимость производящего функционала $\left.Z[j, J]\right|_{j=0}$ от точки нормировки $\mu$ при фиксированном $e_{0}$, поскольку после перемасштабирования суперполей материи $\phi \rightarrow Z^{-1 / 2} \phi$ от $\mu$ будет зависеть $\ln Z$ в аномальном вкладе. Другими словами, если имеет место теорема Адлера-Бардина, то мера в функциональном интеграле будет давать вклад, зависящий от точки нормировки. Поэтому в этом случае функции $\beta$ и $\tilde{\beta}$ уже не будут связаны между собой соотношением (35). Это и объясняет полученное различие этих функций в двухпетлевом приближении, которого не должно быть, если формула (35) имеет место. Здесь еше раз необходимо подчеркнуть, что вопрос о корректности использования голоморфной нормировки в данной работе не обсуждается.

Зависимость производяшего функционала $\left.Z[j, J]\right|_{j=0}$ от точки нормировки отсутствует для канонической нормировки суперполей материи, и тогда в двухпетлевом приближении функции $\beta$ и $\tilde{\beta}$ действительно оказываются одинаковыми. Однако в этом случае теорема Адлера-Бардина не имеет место и проблемы аномалий также не возникает.

5. Противоречивость метода размерной редукции, отмеченная в работе [28], при вычислениях с использованием техники суперграфов без каких-либо дополнительных предположений приводит к нулевым результатам для сумм диаграмм, определяющих аксиальную аномалию и аномалию Кониши, что, в свою очередь, ведет к появлению проблемы аномалий. При этом необходимо заметить, что дополнительных условий типа $\operatorname{tr}(A B) \neq \operatorname{tr}(B A)$, позволяюших получить правильный ответ для аксиальной аномалии, в явно суперсимметричной технике вычислений наложить не удается.

K сожалению, проведенное исследование пока ограничено абелевым случаем. Для суперсимметричной теории Янга-Миллса вычисления с использованием метода высших ковариантных производных представляют большую сложность, поскольку введение в теорию слагаемого с высшими ковариантны.ми производными [40] сушественно усложняет форму действия. В этом случае значительно упростить ситуацию может замена высших ковариантных производных на обычные производные. При этом, конечно, будет нарушена калибровочная инвариантность, что может привести к нарушению тождеств Уорда некоторыми локальными неинвариантными слагаемыми. Тем не менее выбор специальной схемы вычитаний позволяет восстановить калибровочную инвариантность и использовать при вычислениях неинвариантные регуляризации [41], [42]. Для абелевых суперсимметричных теорий такая схема построена в работе [43], а для неабелевых - в работе [44]. 


\section{ПРИЛОЖЕНИЕ}

\section{Вычисление суммы диаграмм с контрчленными вставками во всех порядках теории возмущений}

Для того чтобы доказать равенство (69), необходимо рассмотреть одночастично-неприводимые диаграммы с контрчленными вставками. Рассмотрим вначале все такие диаграммы, содержащие только одну петлю суперполей материи и не имеющие внутренних $V$-линий. Такие вклады могут быть вычислены точно. Действительно, несложно убедиться в том, что эффективная линия

$$
\overline{\bar{x}}=\bar{x}+\frac{x}{x}+\cdots,
$$

где крестиком обозначена контрчленная вставка, соответствует следующим пропагатоpam:

$$
\frac{1}{Z}\left(\begin{array}{cccc}
0 & -\frac{\bar{D}^{2} D^{2}}{16\left(\partial^{2}+m^{2} / Z^{2}\right)} & \frac{m \bar{D}^{2}}{4 Z\left(\partial^{2}+m^{2} / Z^{2}\right)} & 0 \\
-\frac{D^{2} \bar{D}^{2}}{16\left(\partial^{2}+m^{2} / Z^{2}\right)} & 0 & 0 & \frac{m D^{2}}{4 Z\left(\partial^{2}+m^{2} / Z^{2}\right)} \\
\frac{m \bar{D}^{2}}{4 Z\left(\partial^{2}+m^{2} / Z^{2}\right)} & 0 & 0 & \frac{\bar{D}^{2} D^{2}}{16\left(\partial^{2}+m^{2} / Z^{2}\right)} \\
0 & \frac{m D^{2}}{4 Z\left(\partial^{2}+m^{2} / Z^{2}\right)} & \frac{D^{2} \bar{D}^{2}}{16\left(\partial^{2}+m^{2} / Z^{2}\right)} & 0
\end{array}\right) .
$$

При этом $m=0$ для линий, соответствующих полям $\phi$ и $\tilde{\phi}$, и $m=M$ для полей Паули-Вилларса (первая строка в этой матрище соответствует пропагаторам $\phi-\phi, \phi-\phi^{*}$, $\phi-\tilde{\phi}, \phi-\tilde{\phi}^{*}$, вторая строка - пропагаторам $\phi^{*}-\phi, \phi^{*}-\phi^{*}, \phi^{*}-\tilde{\phi}, \phi^{*}-\tilde{\phi}^{*}$ и т.д.).

Подставим пропагаторы (74) в диаграммы

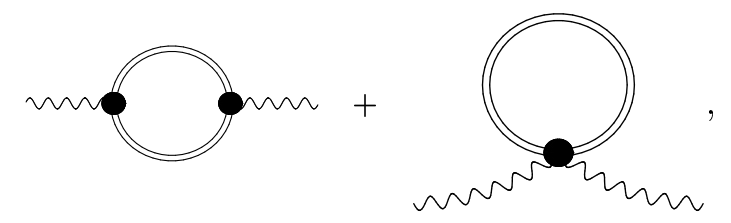

где кружками обозначены эффективные вершины

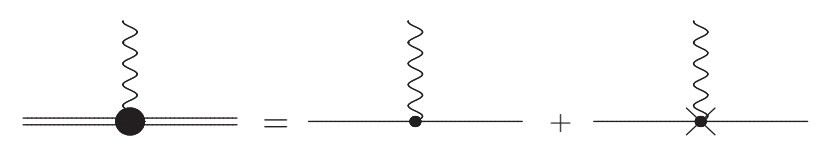

и




которые пропорциональны

$$
Z\left(\begin{array}{cccc}
0 & 1 & 0 & 0 \\
1 & 0 & 0 & 0 \\
0 & 0 & 0 & -1 \\
0 & 0 & -1 & 0
\end{array}\right) \text { и } Z\left(\begin{array}{llll}
0 & 1 & 0 & 0 \\
1 & 0 & 0 & 0 \\
0 & 0 & 0 & 1 \\
0 & 0 & 1 & 0
\end{array}\right)
$$

соответственно. Тогда достаточно очевидно, что результат для диаграмм (75) будет отличаться от результата для обычных однопетлевых диаграмм

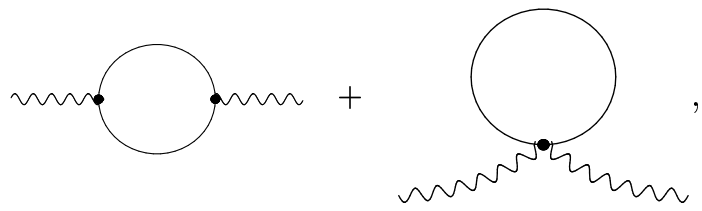

только заменой $M \rightarrow M / Z$ и оказывается равным

$$
\begin{aligned}
-\frac{i}{2} \operatorname{Re} & \int d^{2} \theta \frac{d^{4} p}{(2 \pi)^{2}} W_{a}(p, \theta) C^{a b} W_{b}(-p, \theta) \times \\
& \times \int \frac{d^{4} k}{(2 \pi)^{2}}\left(\frac{1}{k^{2}(k+p)^{2}}-\sum_{i} c_{i} \frac{1}{\left(k^{2}-M_{i}^{2} / Z^{2}\right)\left((k+p)^{2}-M_{i}^{2} / Z^{2}\right)}\right) .
\end{aligned}
$$

После вычитания из этого выражения однопетлевого результата для диаграмм (78) (однопетлевые диаграммы не содержат контрчленных вставок) для вклада суммы диаграмм с контрчленными вставками получается следующий результат:

$$
\begin{aligned}
-\frac{i}{2} \operatorname{Re} & \int d^{2} \theta \frac{d^{4} p}{(2 \pi)^{2}} W_{a}(p, \theta) C^{a b} W_{b}(-p, \theta) \times \\
& \times \sum_{i} c_{i} \int \frac{d^{4} k}{(2 \pi)^{2}}\left(\frac{1}{\left(k^{2}-M_{i}^{2}\right)\left((k+p)^{2}-M_{i}^{2}\right)}-\right. \\
& \left.-\frac{1}{\left(k^{2}-M_{i}^{2} / Z^{2}\right)\left((k+p)^{2}-M_{i}^{2} / Z^{2}\right)}\right) .
\end{aligned}
$$

Совершая поворот Вика и принимая во внимание, что

$$
\begin{aligned}
\frac{1}{2} \int \frac{d^{4} k}{(2 \pi)^{4}} & \frac{1}{\left(k^{2}+M^{2} / Z^{2}\right)\left((k+p)^{2}+M^{2} / Z^{2}\right)}- \\
& -\frac{1}{2} \int \frac{d^{4} k}{(2 \pi)^{4}} \frac{1}{\left(k^{2}+M^{2}\right)\left((k+p)^{2}+M^{2}\right)}= \\
= & \frac{1}{16 \pi^{2}}\left(-\ln \frac{M}{p Z}-\sqrt{1+\frac{4 M^{2}}{p^{2} Z^{2}}} \operatorname{arcth} \sqrt{\frac{p^{2}}{4 M^{2} / Z^{2}+p^{2}}}+\right. \\
& \left.+\ln \frac{M}{p}+\sqrt{1+\frac{4 M^{2}}{p^{2}}} \operatorname{arcth} \sqrt{\frac{p^{2}}{4 M^{2}+p^{2}}}\right)=\frac{1}{16 \pi^{2}} \ln Z+o(1),
\end{aligned}
$$


мы получаем, что сумма одночастично-неприводимых диаграмм с контрчленными вставками для полей материи может быть записана в виде

$$
-\ln Z \frac{1}{16 \pi^{2}} \operatorname{Re} \int d^{4} x d^{2} \theta W_{a} C^{a b} W_{b}+\text { конечные члены, }
$$

что в терминах производящего функционала может быть записано в виде (69).

На следуюшем шаге необходимо рассмотреть диаграммы, содержашие внутренние линии суперполя $V$. Как и в рассмотренном выше случае, несложно убедиться, что точная сумма диаграмм, включающая диаграммы с контрчленными вставками, будет отличаться от точной суммы диаграмм без контрчленных вставок заменой $M \rightarrow M / Z$. Однако при наличии внутренней линии суперполя $V$ будет существовать конечное предельное значение суммы диаграмм, содержаших поля Паули-Вилларса, при $M \rightarrow \infty$. Поэтому в пределе $M \rightarrow \infty$ сумма диаграмм с учетом контрчленных вставок окажется равной сумме обычных диаграмм. Следовательно, “чистый” вклад диаграмм с контрчленными вставками, имеющих внутреннюю линию суперполя $V$, оказывается равным нулю. Это, в свою очередь, означает, что результат (82) является точным во всех порядках теории возмушений.

Благодарности. Автор выражает глубокую благодарность В. А. Новикову, П. И. Пронину, А. А. Славнову и А. А. Солошенко за полезные обсуждения.

\section{Список литературы}

[1] S. Ferrara, B. Zumino. Nucl. Phys. B. 1975. V. 87. P. 207.

[2] T. E. Clark, O. Piquet, K. Sibold. Nucl. Phys. B. 1978. V. 143. P. 445.

[3] O. Piquet, K. Sibold. Nucl. Phys. B. 1982. V. 196. P. 428.

[4] O. Piquet, K. Sibold. Nucl. Phys. B. 1982. V. 196. P. 447.

[5] S. L. Adler, W. A. Bardeen. Phys. Rev. 1969. V. 182. P. 1517.

[6] А. А. Славнов, Л. Д. Фаддеев. Введение в квантовую теорию калибровочных полей. М.: Наука, 1988.

[7] S. L. Adler, J. C. Collins, A. Duncan. Phys. Rev. D. 1977. V. 15. P. 1712.

[8] V. A. Novikov, M. A. Shifman, A. I. Vainstein, V. I. Zakharov. Phys. Lett. B. 1985. V. 157. P. 169 .

[9] P. S. Howe, K. S. Stelle, P. S. West. Phys. Lett. B. 1983. V. 124. P. 55.

[10] O. V. Tarasov, V. A. Vladimirov. Phys. Lett. B. 1980. V. 96. P. 94.

[11] M. T. Grisaru, M. Rocek, W. Siegel. Phys. Rev. Lett. 1980. V. 45. P. 1063.

[12] W. Caswell, D. Zanon. Phys. Lett. B. 1980. V. 100. P. 152.

[13] M. Shifman, A. Vainstein. Nucl. Phys. B. 1986. V. 277. P. 456.

[14] K. Konishi. Phys. Lett. B. 1984. V. 135. P. 439.

[15] T. E. Clark, O. Piquet, K. Sibold. Nucl. Phys. B. 1979. V. 159. P. 1.

[16] V. Novikov, M. Shifman, A. Vainstein, V. Zakharov. Phys. Lett. B. 1985. V. 166. P. 329.

[17] L. Avdeev, O. Tarasov. Phys. Lett. B. 1982. V. 112. P. 356.

[18] I. Jack, D. Jones, C. North. Nucl. Phys. B. 1996. V. 473. P. 308.

[19] I. Jack, D. Jones, C. North. Phys. Lett. B. 1996. V. 386. P. 138.

[20] I. Jack, D. R. T. Jones, C. G. North. Nucl. Phys. B. 1997. V. 486. P. 479. 
[21] N. Arkani-Hamed, H. Mirayama. JHEP. 2000. V. 0006. P. 030; hep-th/9707133.

[22] А. А. Славнов. ТМФ. 1975. Т. 23. С. 3.

[23] T. Bakeyev, A. Slavnov. Mod. Phys. Lett. A. 1996. V. 11. P. 1539.

[24] P. Pronin, K. Stepanyantz. Phys. Lett. B. 1997. V. 414. P. 117.

[25] A. Soloshenko, K. Stepanyantz. Two-loop renormalization of $N=1$ supersymmetric electrodynamics, regularized by higher derivatives. hep-th/0203118.

[26] A. А. Солошенко, К. В. Степаньянц. ТМФ. 2003. Т. 134. С. 430.

[27] W. Siegel. Phys. Lett. B. 1979. V. 84. P. 193.

[28] W. Siegel. Phys. Lett. B. 1980. V. 94. P. 37.

[29] G. 't Hooft, M. Veltman. Nucl. Phys. B. 1972. V. 44. P. 189.

[30] H. Nicolai, P. K. Townsend. Phys. Lett. B. 1980. V. 93. P. 111.

[31] D. R. T. Jones, J. P. Leveille. Nucl. Phys. B. 1982. V. 206. P. 473.

[32] П. Уэст. Введение в суперсимметрию и супергравитацию. М.: Мир, 1989.

[33] A. A. Soloshenko, K. V. Stepanyantz. Three-loop $\beta$-function for $N=1$ supersymmetric electrodynamics, regularized by higher derivatives. hep-th/0304083.

[34] К. Иииксон, Ж.-Б. Зюбер. Квантовая теория поля. М.: Мир, 1984.

[35] Джс. Коллинз. Перенормировка. Новосибирск: ИО НФМИ, 2000.

[36] A. А. Солошенко, K. В. Степаньяни. ТМФ. 2002. Т. 131. С. 135.

[37] I. Jack, D. Jones. Regularisation of supersymmetric theories. hep-ph/9707278.

[38] G. 't Hooft. Nucl. Phys. B. 1973. V. 61. P. 455.

[39] R. Bertlmann. Anomalies in Quantum Field Theory. Oxford: Clarendon, 1996.

[40] P. West. Nucl. Phys. B. 1986. V. 268. P. 113.

[41] A. A. Slavnov. Phys. Lett. B. 2001. V. 518. P. 195.

[42] A. А. Славнов. ТМф. 2002. Т. 130. С. 3.

[43] A. А. Славнов, K. В. Степаньяни. ТМФ. 2003. Т. 135. № 2. C. 265 ; hep-th/0208006.

[44] А. А. Славнов, К. В. Степаньяни. ТМФ. 2004. Т. 139. № 2. С. 179; hep-th/0305128.

Поступила в редакцию 30.I.2003 г., после доработки 6.VI.2003 г. 